\title{
Commentary: Light, and maybe less bacteria, at the end of the tunnel?
}

\author{
Antonio G. Cabrera, MD, ${ }^{\mathrm{a}}$ Mubbasheer Ahmed, MD, ${ }^{\mathrm{b}}$ and Paul A. Checchia, MD, FCCM, FACC ${ }^{\mathrm{b}}$
}

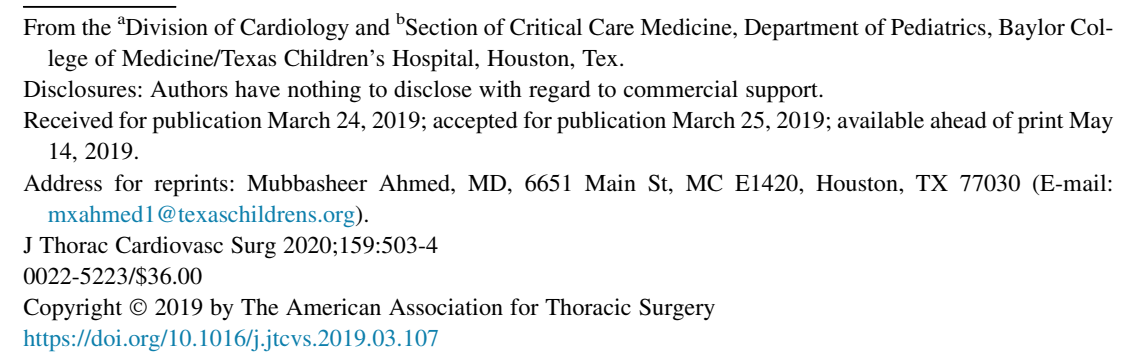

Prevalence of heart failure continues to increase with an estimated 8 million to be affected by $2030 .^{1}$ As organ demand outpaces supply, durable mechanical support implantation has revolutionized the care of these patients. ${ }^{1,2}$ Although thrombosis and bleeding continue to be the main complication, infections affect $18 \%$ to $59 \%$ of left ventricular assist device (LVAD) cases. ${ }^{1}$ Driveline infections (DLIs) are the most prevalent (14\%-48\%) among LVAD-associated infections. ${ }^{1-3}$ A retrospective analysis of 247 patients found $32 \%$ of their cohort affected by infections with $47 \%$ due to DLIs. ${ }^{4}$ LVAD infections lead to decreased quality of life, increased readmission rates, and increased mortality. ${ }^{1-3}$

Driveline colonization with subsequent tissue invasion is a crucial factor in ventricular assist device (VAD)-associated infections. A murine model concluded bacterial ability to form biofilms was a critical virulence determinant in facilitating DLIs. ${ }^{3,5}$ Drivelines provide passage for bacterial invasion, and their structure enables biofilm formation. ${ }^{2}$ VAD manufacturers have made adaptations to chemical properties and physical architecture of drivelines in an attempt to provide better anchoring of the driveline within the subcutaneous tunnel and to create a less hospitable environment for micro-organisms to form biofilms.

We read with great interest the work of $\mathrm{Qu}$ and colleagues ${ }^{6}$ published in this edition of the Journal, titled "Biofilm Formation and Migration on Ventricular Assist Device Driveline." Qu and colleagues ${ }^{6}$ have provided a meticulous analysis of driveline characteristics and its relation to biofilm formation via in vitro experiments and examination of 2 explanted drivelines. The group provides valuable conclusions to aid understanding of driveline biofilm formation: (1) Biofilm forms on both smooth and velour driveline components; (2) the microscopic velour

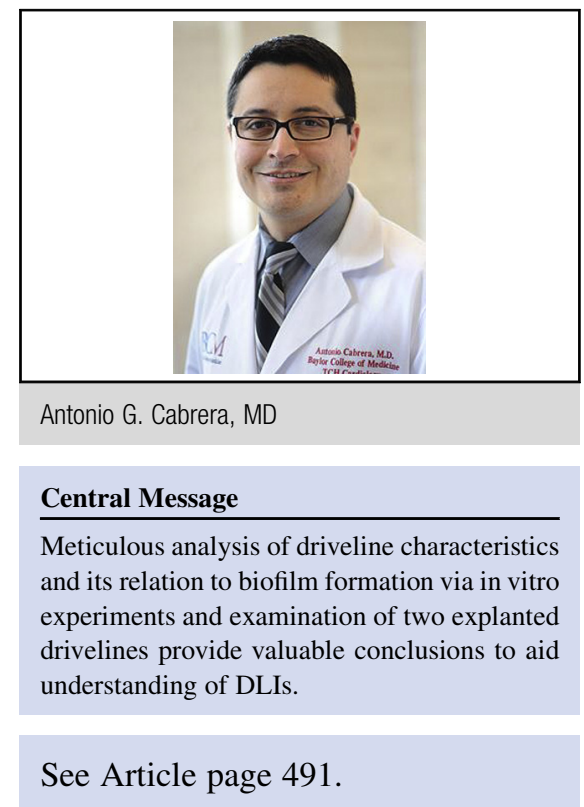

network and the presence of silicone adhesives foster biofilm development; (3) biofilm migration is affected by tissue environment with organism-specific patterns; and (4) explanted drivelines show the presence of micro-gaps between tissue and velour that may facilitate biofilm establishment.

The findings in the article point to both physical (architectural scaffold of velour) and chemical (silicone-based adhesives) attributes that provide possible sites for modification to reduce infectious risk in patients with VADs. In addition, the study of explanted VADs found inadequate tissue in-growth into the velour matrix, and thus creating micro-gaps between tissue and driveline. Obliteration of the microgaps by boosting tissue growth may further reduce infectious complications.

This notable analysis must be balanced by the limitations of the study, in particular the in vitro nature of examination. The formation of biofilms was evaluated in the absence of a host immunologic response and host comorbidities (eg, nutritional status, organ injury, obesity, fluid pockets). This constraint is highlighted by the findings in the 2 explanted drivelines, which solely found a low density (3-4 colony-forming unit $/ \mathrm{mm}^{2}$ ) of Staphylococcus epidermidis at the exit site of one of the drivelines. Clearly, the host environment complicates our attempts at a neat mechanistic understanding. 
Until elimination of driveline-based energy transfer systems takes place, a mechanistic insight into biofilm formation and methods to combat biofilm development is vital in improving morbidity and mortality in patients with VADs.

\section{References}

1. Hernandez GA, Breton JDN, Chaparro SV. Driveline infection in ventricular assist devices and its implication in the present era of destination therapy. Open J Cardiovasc Surg. 2017;9:1179065217714216.

2. Leuck AM. Left ventricular assist device driveline infections: recent advances and future goals. J Thorac Dis. 2015;7:2151-7.
3. Hieda M, Sata M, Nakatani T. The importance of the management of infectious complications for patients with left ventricular assist device. Healthcare (Basel). 2015;3:750-6.

4. Nienaber JJ, Kusne S, Riaz T, Walker RC, Baddour LM, Wright AJ, et al. Clinical manifestations and management of left ventricular assist device-associated infections. Clin Infect Dis. 2013;57:1438-48.

5. Toba FA, Akashi H, Arrecubieta C, Lowy FD. Role of biofilm in Staphylococcus aureus and Staphylococcus epidermidis ventricular assist device driveline infections. J Thorac Cardiovasc Surg. 2011;141: 1259-64.

6. Qu Y, McGiffin D, Kure C, Ozcelik B, Fraser J, Thissen H, et al. Biofilm formation and migration on ventricular assist device drivelines. J Thorac Cardiovasc Surg. 2020;159:491-502.e2. 